\title{
Research on Space Debris Removal Region Selection and Removal Strategy
}

\author{
Ningwei $\mathrm{Wu}^{1, \mathrm{a},{ }^{*} \text {, Yasheng Zhang }}{ }^{2, \mathrm{~b}}$, and Xumin Song ${ }^{3, \mathrm{c}}$ \\ ${ }^{1}$ Department of Graduate Management, Equipment Academy of PLA, Beijing 101416, China. \\ ${ }^{2}$ Department of Space Equipment, Equipment Academy of PLA, Beijing 101416, China \\ ${ }^{3}$ Department of Space Command, Equipment Academy of PLA, Beijing 101416, China \\ a Corresponding author: 1054867214@qq.combxianhao168@sina.com673517162@qq.com
}

Keywords: Space debris removal, Distribution and evolution rule, Removal region selection Removal strategy.

\begin{abstract}
This paper uses almost the latest SSR (by March 3, 2017) to present nearly 18,000 in-orbit objects which can be detectable by the ground with the size greater than $10 \mathrm{~cm}$. After the statistical comparison and requirement analysis, this paper selects SSO orbit and its perimeter zone to be the target region to carry out space debris removal operation. By using the historical TLE data to analyze the long-term evolution rule of the object in these regions, some feasible strategies are given in order to remove more debris with less energy.
\end{abstract}

\section{Introduction}

Since the Soviet Union launched the world's first man-made earth satellite in 1957, thousands of times of the space launch mission makes the number of spacecraft keeps increasing in space. Fig 1 shows all the space objects in space (plotted by MATLAB from the TLE data by March 3, 2017).

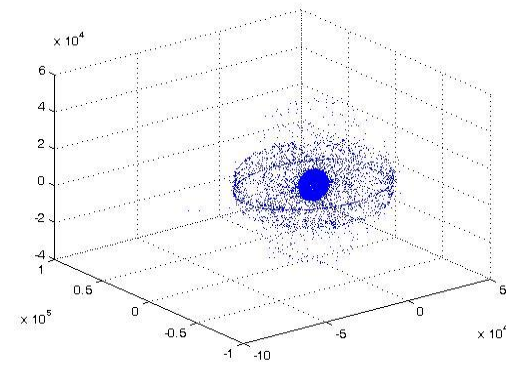

Figure 1. Distribution exhibition

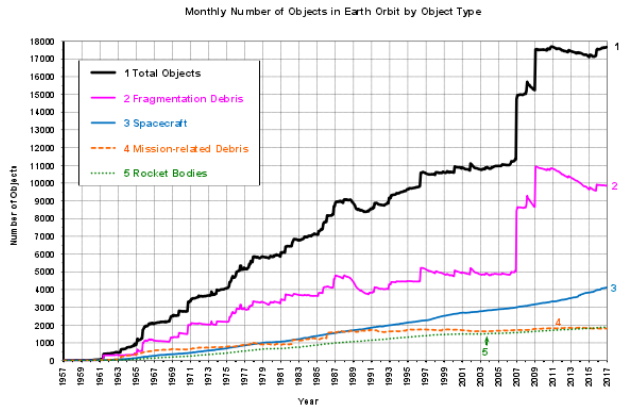

Figure 2. Change in object number

Fig 2 shows the increase in the number of space objects provided by NASA ${ }^{\left[{ }^{]}\right.}$and as a result, the requirement for starting the debris removal as soon as possible is becoming more and more urgent.

Esmiller [4] and the State Key Laboratory of Laser Propulsion \& Application [5] have carried out the research on the removal in laser way at ground. The reason why the way has not yet been put into the application is the difficulty in the target positioning accuracy and the high cost.

With the mature rendezvous technology and in-orbit operation skills, many scholars put forward a solution in the space-based platform removal way. However, most of the current space debris removal plans are still in conceptual design. One of the reasons is that the space-based debris removal involves multi-target rendezvous, and its fuel consumption is significantly higher than the current capacity of spacecraft nowadays. To better solve this problem, many scholars are using various optimization algorithms to conduct an in-depth study on the optimal rendezvous issues. Representatives have Yazhong Luo [6], Carter [7] et al.

This paper presents a method to solve the debris problem by selecting the removal region with the debris distribution statistics first and giving a new removal strategy via using the space debris orbital evolution rule so that we can maneuver less to save energy and remove more debris. 


\section{Removal Region Selection}

The space objectives are divided into the following four categories: on-orbit payload, functional failure load, rocket body and other space debris. Except the on-orbit payload, the failure load, the rocket and other debris are collectively referred to as space debris, which is often called space junk.

As of March 3, 2017, the total number of space objects that could be observed was 17,995, including 17761 objects moving around the Earth, 229 objects that had flowed out of the Earth's gravitational field and 5 had been docked with other objects. These 17761 space objects include 1537 on- orbit payload, 2871 the failure load, 2053 the rocket body and 11300 other debris. The proportion of each part is shown in Fig 3:
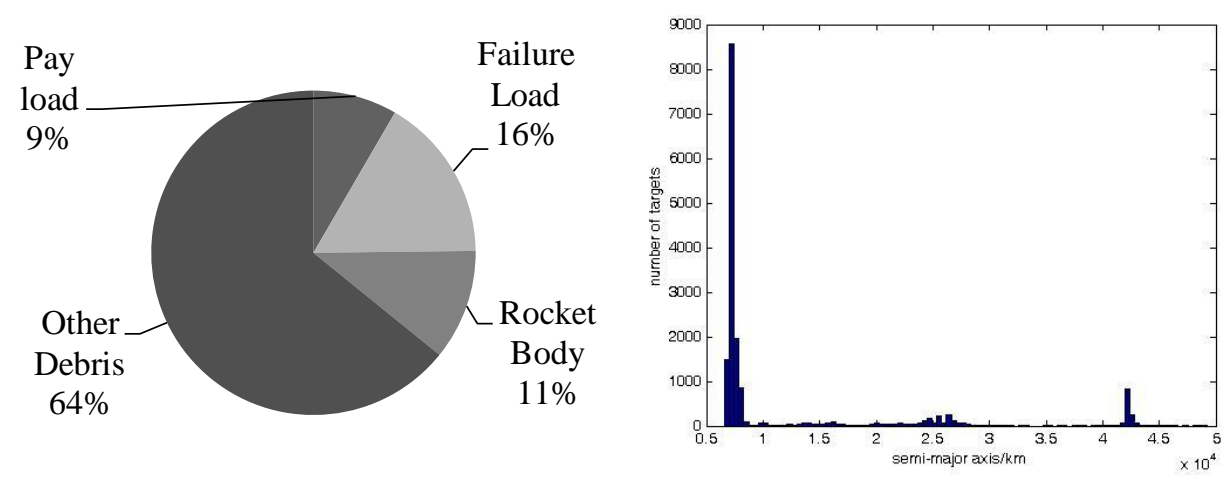

Figure 3. Proportion of different objects Figure 4. All semi-major axis distribution

The space objects are divided into the following four categories: Low Earth orbit (LEO), Medium Earth Orbit (MEO), Geostationary Orbit (GEO) and Highly Elliptical Orbit (HEO). According to the space objects clustering shown in Fig 4, we can see the LEO objects are of the semi-major axis less than $8500 \mathrm{~km}$. In the nearly 18,000 space objects, there are more than 14,000 LEO space objects, accounting for more than $75 \%$, which is the main destination of the space objects, and is also the main gathering area of space debris, for there are nearly 11000 space debris. In another word, this area is the primary area where space debris removal operation needs to consider.

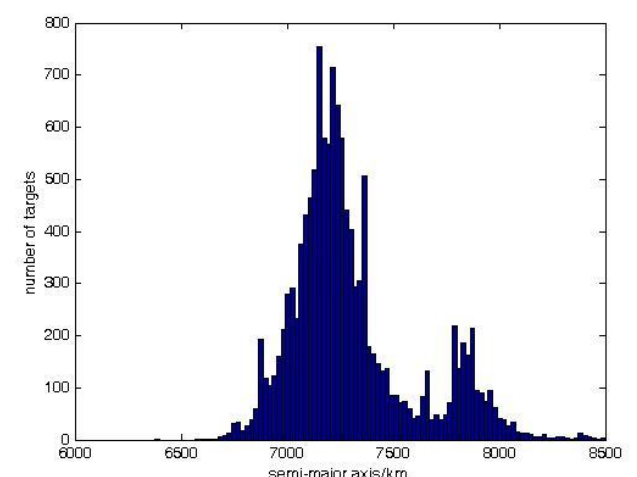

Figure 5. LEO semi-major axis distribution

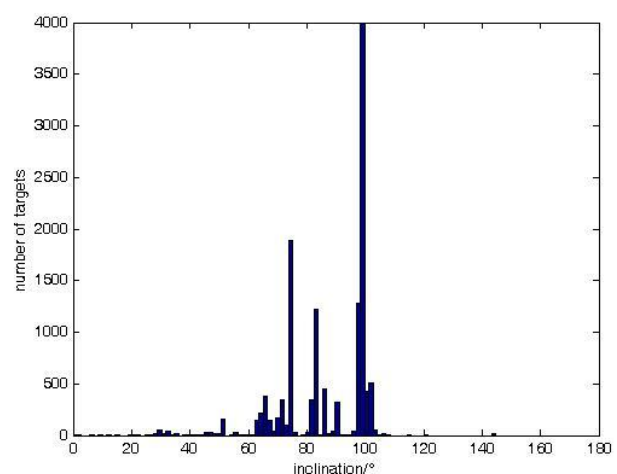

Figure 6. LEO inclination distribution

It can be seen from Fig 5, LEO space objects which are concentrated on the semi-major axis between $7000-7500 \mathrm{~km}$, as the orbital altitude is $600 \mathrm{~km}-1000 \mathrm{~km}$, accounting for more than $60 \%$. Especially, we have the densest distribution in the orbital altitude of $800 \mathrm{~km}$. It can be seen from Fig 6 that the orbital inclination is concentrated in the range of $80-120^{\circ}$ and the proportion is close to $65 \%$. That is to say, the Low-Orbit space objects are concentrated in this inclination range, which is the solar synchronous orbit (SSO) and its surrounding areas. Because of its characteristic of sunoriented, SSO is beyond doubt to be an important gathering place which attracts many payloads, and also becomes the most crowed area for space debris, for more than $80 \%$ of the objects in this area are space debris. 


\section{SSO Evolution Rule Analysis}

The distribution of the space objects mentioned above reflects only the current situation of the orbital distribution of space objects in a certain epoch. The orbit is changing in real time due to the perturbation. The space objects in the SSO orbit are mainly affected by the non-spherical perturbation of the Earth, atmospheric drag perturbation, solar radiation pressure perturbation, three-body perturbation and other perturbation.

TLE data is the information data of the catalog space object which is regularly updated by the North American Air Defense Command. The data is based on the observed value and the periodic perturbation term is removed by a particular way, whereby the average orbital data of the space object can be obtained. The information from TLE can factually and intuitively reflect the long-term evolution in the orbit of all the objects. Because of the chronicity of debris removal, in this paper, four objects are selected with the NORAD numbers of 815, 4973, 2413 and 3591. Using the historical TLE data from March 3, 2016 to March 3, 2017, the evolution rule of the four main orbital elements (semi-major axis, eccentricity, orbital inclination and RAAN) are shown below from Fig 7-Fig 10.

Table 1. Part of the current orbital elements of four objects selected

\begin{tabular}{ccccc}
\hline Name & NORAD & $\mathrm{a} / \mathrm{km}$ & $\mathrm{e}$ & $\mathrm{i} /{ }^{\circ}$ \\
\hline THOR AGENA D Rocket Body & 815 & 7179 & 0.001411 & 99.86 \\
THOR AGENA D Debris & 4973 & 7277 & 0.009564 & 100 \\
SCOUT A Rocket Body & 2413 & 7445 & 0.00392 & 88.85 \\
SCOUT A Debris & 3591 & 7263 & 0.00878 & 90.15 \\
\hline
\end{tabular}
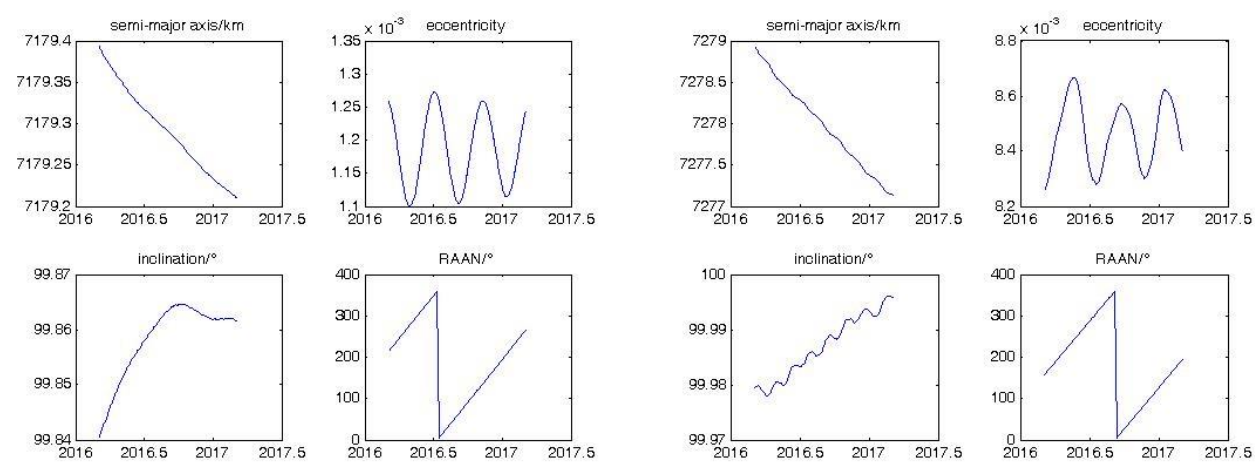

Figure 7. NORAD 815
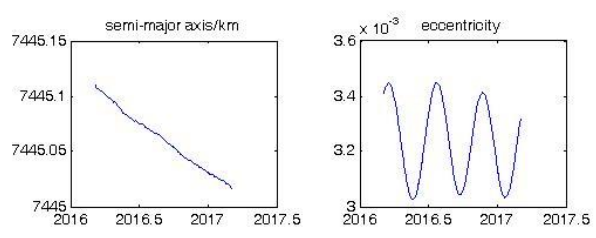

Figure 8. NORAD 4973
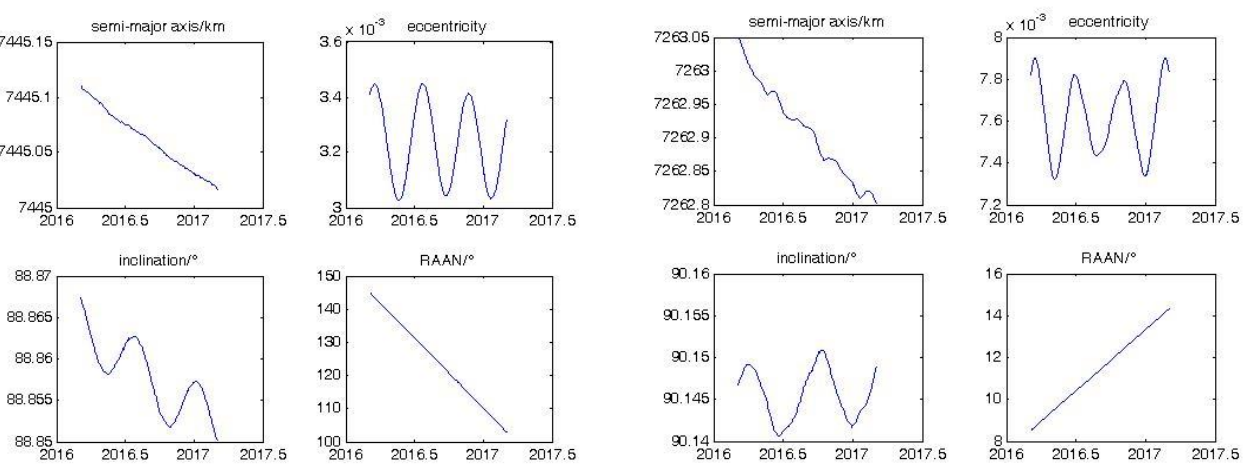

Figure 9. NORAD 2413

Figure 10. NORAD 3591

As can be seen from the Fig 7-Fig 10, the semi-major axis of the object continues to decline, the eccentricity is cyclically oscillating, and the orbital inclination changes differently. The above three elements appears to be completely different with the change of different objects, but its overall range changes slightly. In another aspect, the four RAAN of the four objects show a significant periodical and linear change. All of the evolution rule can be used for us, which will be discussed next. 


\section{Space Debris Removal Strategy}

The above-mentioned distribution clearly shows that the target area of space debris removal is SSO and its surrounding region. And the space objects in this region also show the useful evolution rule which can be exploited with the debris removal strategy.

We all know that most fuel consumption is caused by the orbital maneuver which aims to change the difference between two planes. Due to the semi-major axis and eccentricity have little effect on the difference, so we assume the two orbits are on the same celestial sphere. The angle between two planes is shown in Fig 11 below:

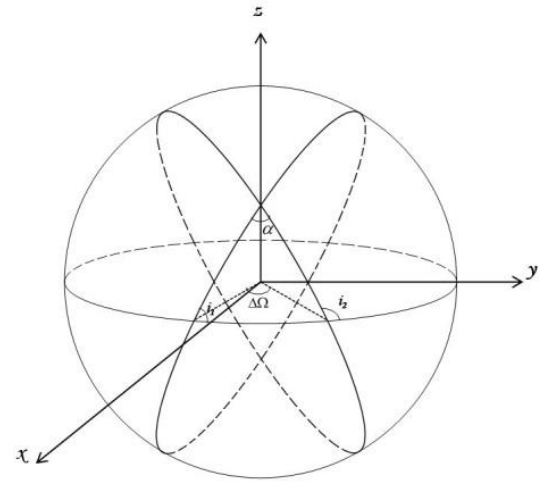

Figure 11. Schematic diagram of two different orbital planes on celestial sphere

From Fig 11 above, we can use the spherical triangle formula to calculate the degree between two different orbital planes as shown in Equation 1:

$$
\alpha=\arccos \left(\operatorname{cosi}_{1} \operatorname{cosi} i_{2}+\operatorname{sini}_{1} \operatorname{sini}_{1} \cos \Delta \Omega\right)
$$

We can see that the difference is mainly affected by the inclination and RAAN. Fig 7-Fig 10 shows that the orbital inclination does not change a lot, the difference between two orbital planes is mainly affected by the difference between RAANs. The changes in RAANs follow strictly periodicity which can be used into the removal strategy.

\section{Strategy 1}

In this region, if the orbit of the object is very close to each other, the change of their RAAN is basically synchronized, so that we can speculate that the orbit which has almost the same orbit elements can be synchronized for a long time, such as 815 and 4973, which can be used by the space debris removal. The space debris remover can be designed to operate in the same area with the debris and evolve along with other debris, which makes the remover work approximate in coplanar throughout the removal process.

We screened 10 TLE data which have the orbital elements almost the same. Fig 12 shows the evolution results during a year (2016/03/03-2017/03/03, the middle figure is the evolution six months later after the initial moment, Fig 13 and 14 below have the same explanation). This strategy can greatly reduce the cost of energy consumption in space maneuvering.
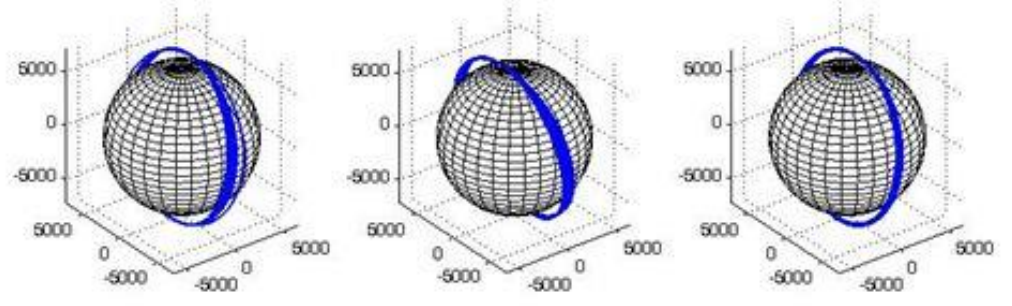

\section{Strategy 2}

Figure 12. Same evolution trend

At the same time, we can also see that if the space inclination of the orbital inclination is slightly different, it will cause the different speed of the change of RAAN, and their orbit can be seriously dispersed in the long evolution. We screened 20 TLE data which have the orbital elements just a little 
bit difference with each other at the initial moment, as a result, after the evolution of a year, their orbit are seriously dispersed. Fig 13 shows the change below:
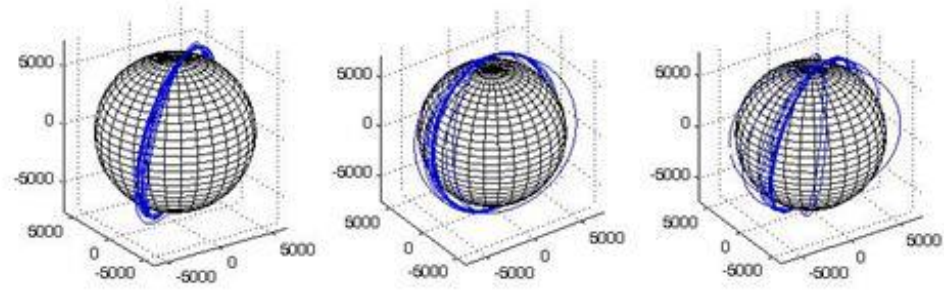

Figure 13. Decentralized trend

But in another word, due to the change of RAAN has the smart periodicity, this feature can also be used. We screened 26 TLE data whose RAANs have a great difference with each other at the initial moment, after the evolution of a year, as a result, their orbit are polymeric. Fig 14 shows the feather.
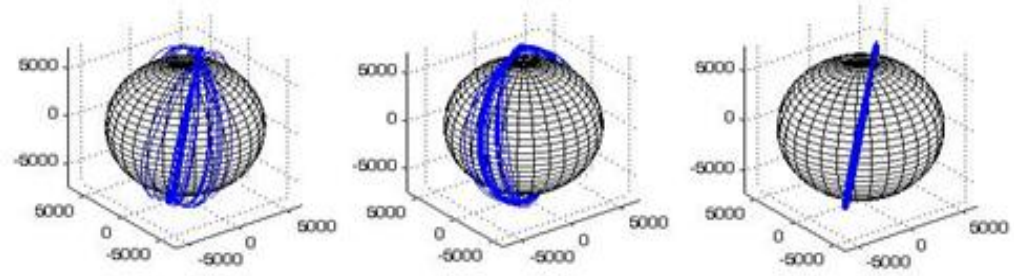

Figure 14. Aggregation trend

This feature can be used in the planning of space debris removal tasks. In the long-term mission the remover can just wait for the object to gather together and start its mission by maneuvering in the condition of coplanar, which also saves the energy.

\section{Conclusion}

With the continuous increase of space objects and the blowout of space missions, especially the multi-star launch mission has become the mainstream, how to use the distribution and evolution rule of space objects for the future spaceflight missions will be a continuous research hotspot. Except the problem of space debris removal, the safety of the future spacecraft operation and the problem of space traffic control will also be the internal driving force of the research on this issue. Based on the latest data, this paper makes a systematic study on the distribution and evolution rule of space objects. At the same time, the strategies of solving the problem of space debris removal are given by using the evolution rule of the change of RAAN. It is believed that with the continuous improvement of space object observation methods, the continuous development of space dynamics and the continuous progress of spacecraft carrying capacity, the space environment will continue to provide an excellent environment for human beings.

\section{Acknowledgments}

Here, I would like to thank my tutors Yasheng Zhang and Xumin Song, I will express my respect to Professor Li Zhi and all the tutors in his research group in which I began my first step in scientific research. At the same time, I would like to praise my roommate and classmates in my study life who encourage and help me to overcome difficulties when I lost my direction. Sincerely thank you all!

\section{References}

[1]. The Satellite Situation Report [DB/OL]. http://www. Space-track.org, 2017-03-03.

[2]. Orbital Debris Quarterly News, Volume 21, Issue 1[EB/OL].https://orbitaldebris.jsc.nasa.gov, 2017-02 
[3]. Esmiller B, Jacquelard C, Eckel H A, et al. Space Debris Removal by Ground-based Lasers: Main Conclusions of the European Project CLEANSPACE[J]. Applied Optics, 2014, 53(31):I45.

[4]. Chang H, Jin X, Yanji H, Xiuqian Li. Construction and Simulation for Space Debris Removal Process by Ground-based Lasers [J]. Journal of Aeronautical, 2012,06: 994-1001

[5]. Zhang J, Parks G T, Luo Y, et al. Multi-spacecraft Refueling Optimization Considering the J2 Perturbation and Window Constraints [J]. Journal of Guidance Control \& Dynamics, 2015, 37(1):111-122.

[6]. Carter T, Humi M. Fuel-optimal rendezvous near a point in general Keplerian orbit [J]. Journal of Guidance Control \& Dynamics, 2012, 10(6):567-573. 